\title{
ON A LEMMA OF MILUTIN \\ CONCERNING AVERAGING OPERATORS IN CONTINUOUS FUNCTION SPACES
}

\begin{abstract}
BY
SEYMOUR Z. DITOR( $\left.{ }^{1}\right)$

Abstract. We show that any infinite compact Hausdorff space $S$ is the continuous image of a totally disconnected compact Hausdorff space $S^{\prime}$, having the same topological weight as $S$, by a map $\varphi$ which admits a regular linear operator of averaging, i.e., a projection of norm one of $C\left(S^{\prime}\right)$ onto $\varphi^{\circ} C(S)$, where $\varphi^{\circ}: C(S) \rightarrow C\left(S^{\prime}\right)$ is the isometric embedding which takes $f \in C(S)$ into $f \circ \varphi$. A corollary of this theorem is that if $S$ is an absolute extensor for totally disconnected spaces, the space $S^{\prime}$ can be taken to be the Cantor space $\{0,1\}^{\mathrm{m}}$, where $\mathrm{m}$ is the topological weight of $S$. This generalizes a result due to Milutin and Petczyński. In addition, we show that for compact metric spaces $S$ and $T$ and any continuous surjection $\varphi: S \rightarrow T$, the operator $u: C(S) \rightarrow C(T)$ is a regular averaging operator for $\varphi$ if and only if $u$ has a representation $u f(t)$ $=\int_{0}^{1} f(\theta(t, x)) d x$ for a suitable function $\theta: T \times[0,1] \rightarrow S$.
\end{abstract}

1. Introduction. In his proof that for uncountable compact metric spaces $S$, all Banach spaces $C(S)$ of continuous (scalar-valued) functions on $S$ with the sup norm are linearly homeomorphic, Milutin [5], [6] establishes the following result:

Milutin's Lemma. There is a continuous map of the Cantor set $K$ onto the unit interval I for which there exists a projection of norm one of $C(K)$ onto $\varphi^{\circ} C(I)$, where $\varphi^{\circ}: C(I) \rightarrow C(K)$ is the isometric embedding which takes $f \in C(I)$ into its composition with $\varphi, \varphi^{\circ}(f)=f \varphi$.

An equivalent formulation of the above is that there is a linear operator $u$ of norm one from $C(K)$ to $C(I)$ with the property that $u(f \varphi)=f$ for all $f \in C(I)$, i.e. $u \varphi^{\circ}=\mathrm{id}_{C(I)}$. Following Pełczyński [7], we call such an operator $u$ a regular linear operator of averaging for $\varphi$. Using this crucial lemma, Pełczyński [7, Theorem 5.6] has shown that the same result holds if the unit interval is replaced by any compact

Received by the editors May 2, 1969, and, in revised form, November 19, 1969.

AMS Subject Classifications. Primary 4725, 4625.

Key Words and Phrases. Averaging operator, extension operator, linear exave, projection operator, regular operatór, continuous function space, Milutin space, totally disconnected space, inverse and direct limits, integral representation of regular averaging and extension operators.

( $\left.{ }^{1}\right)$ This research was supported by NSF Grant GP-5138.

It is part of the author's Ph.D. Dissertation, Linear operators of averaging and extension, written at the University of California at Berkeley in 1968 under the direction of Professor W. G. Bade. 
metric space. In fact, Pełczyński shows that even if $S$ is the product of $\mathfrak{m}>\boldsymbol{\aleph}_{0}$ compact metric spaces, there is a continuous map of the Cantor space $D^{\mathfrak{m}}$ onto $S$ which admits a regular linear operator of averaging, where $D$ is the two-point space $\{0,1\}$. Such a space $S$ which is the continuous image of some Cantor space $D^{\mathfrak{m}}$ by a map which admits a regular averaging operator is called a Milutin space ( $S$, as for all topological spaces mentioned here, is assumed to be compact Hausdorff).

Here we shall prove the following generalization of Milutin's Lemma:

THEOREM 1. Any infinite compact Hausdorff space is the continuous image of a totally disconnected perfect compact Hausdorff space of the same topological weight by a map which admits a regular linear operator of averaging.

By the topological weight (or simply, weight) of a space $S$ we mean, as usual, the smallest cardinal number which is the cardinality of a base for the topology of $S$. That Theorem 1 generalizes Milutin's Lemma (and Pełczyński's extension to compact metric spaces) follows from the well-known characterization (cf. [2, p. 99]) of the Cantor set as a totally disconnected perfect compact metric space and the fact that a compact Hausdorff space is metrizable if and only if its weight is $\leqq \boldsymbol{\aleph}_{0}$.

If $S$ is an absolute extensor for totally disconnected spaces (i.e., if for any totally disconnected space $X$ and closed subspace $A$, any continuous map from $A$ to $S$ can be extended to a continuous map from $X$ to $S$ ), then an easy corollary of Theorem 1 is that $S$ is a Milutin space. Since Michael [4] has shown that any compact metric space, and therefore any product of compact metric spaces, is an absolute extensor for totally disconnected spaces, this corollary includes the above mentioned result of Pełczyński.

The proof of Theorem 1 is in a sense almost "natural" and in the metric case provides a new proof of Milutin's Lemma. The proof of the lemma which is due to Milutin (cf. [7, Lemma 5.5]) depends on an ingenious construction of a map $\varphi: K \rightarrow I$ for which the regular averaging operator $u: C(K) \rightarrow C(I)$ can be defined by a formula, $u f(t)=\int_{0}^{1} f(\theta(t, x)) d x$, for $f \in C(K), t \in I$, where $\theta: I \times I \rightarrow K$ is a Lebesgue measurable function whose definition is tied to that for $\varphi$. In Theorem 2 we show that every regular averaging operator $u: C(S) \rightarrow C(T)$, for compact metric spaces $S$ and $T$, has a representation $u f(t)=\int_{0}^{1} f(\theta(t, x)) d x$ for a suitable function $\theta: T \times I \rightarrow S$, and conversely, every such representation defines a regular averaging operator.

2. Preliminaries. We need two technical lemmas. One of these (Lemma 1) appears in [7] but not quite in the form we require. However, as the proof given in [7] (a partition of unity argument) carries over verbatim to the more general formulation, we shall state this lemma without proof. As for the other result (Lemma 2), although half of it appears in [7] (cf. [8] also) we shall for the sake of completeness give the entire proof. Since both lemmas have application in a more 
general situation and no further effort is required, we shall introduce Pełczyński's idea of a linear exave and state the lemmas in this more general setting.

Definition. Let $S, T$ be compact Hausdorff spaces and $\varphi: S \rightarrow T$ a continuous map. A bounded linear operator $u: C(S) \rightarrow C(T)$ is called a linear exave for $\varphi$ if $\varphi^{\circ} u \varphi^{\circ}=\varphi^{\circ}$. If $\varphi$ is onto (resp. one-to-one), then $u$ is called a linear operator of averaging (resp. extension) for $\varphi . u$ is called regular if $\|u\|=1$ and $u\left(1_{S}\right)=1_{T}$ (where $1_{X}$ is the constant function on $X$ everywhere equal to one).

Note that if $\varphi$ is onto (resp. one-to-one), then $\varphi^{\circ}$ is one-to-one (resp. onto) and $\varphi^{\circ} u \varphi^{\circ}=\varphi^{\circ}$ reduces to $u \varphi^{\circ}=\mathrm{id}_{C(T)}$ (resp. $\left.\varphi^{\circ} u=\mathrm{id}_{C(S)}\right)$. If $S \subset T$ and $\varphi$ is the inclusion map, then $\varphi^{\circ}: C(T) \rightarrow C(S)$ is the restriction operator, $\varphi^{\circ}(g)=g \mid S$, and for each $f \in C(S), u(f)$ is indeed an extension of $f$ to all of $T$, i.e. $u(f) \mid S=\varphi^{\circ} u(f)=f$.

The connection with projections is immediate (cf. [7, §2]): averaging operators correspond to bounded projections onto closed selfadjoint subalgebras ( $\varphi$ admits a linear operator of averaging if and only if there is a bounded projection of $C(S)$ onto $\varphi^{\circ} C(T)$ ); extension operators correspond to bounded projections onto closed ideals ( $\varphi$ admits a linear operator of extension if and only if there is a bounded projection of $C(T)$ onto the null space of $\varphi^{\circ}$, i.e., the ideal of functions which vanish on $\varphi(S))$.

LEMMA 1. Given a continuous map $\varphi: S \rightarrow T$, where $S, T$ are compact Hausdorff spaces, suppose that $\left\{T_{\alpha}\right\}_{\alpha \in A}$ is a family of closed subsets of $T$, that $\left\{U_{\alpha}\right\}_{\alpha \in A}$ is a family of open subsets of $T$ which cover $\varphi(S)$, and that $U_{\alpha} \subset T_{\alpha}$ for all $\alpha \in A$. Suppose further that $\left\{S_{\alpha}\right\}_{\alpha \in A}$ is a family of closed subsets of $S$ such that for all $\alpha \in A, U_{\alpha} \cap \varphi(S)$ $\subset \varphi\left(S_{\alpha}\right) \subset T_{\alpha}$, and that each $\varphi \mid S_{\alpha}: S_{\alpha} \rightarrow T_{\alpha}$ admits a (regular) linear exave. Then $\varphi$ admits a (regular) linear exave.

In what follows we require the idea of an inverse limit system. The basic facts we use can be found in [1, pp. 212-220].

LEMMA 2. Let $\left(\left\{S_{\beta}\right\},\left\{\pi_{\alpha}^{\beta}\right\}\right)$ and $\left(\left\{T_{\beta}\right\},\left\{\theta_{\alpha}^{\beta}\right\}\right)$ be inverse limit systems of compact Hausdorff spaces and continuous maps over the directed set $A$ with each $\pi_{\alpha}^{\beta}$ onto. Let $\left(S,\left\{\pi_{\alpha}\right\}\right)=\operatorname{inv} \lim \left(\left\{S_{\beta}\right\},\left\{\pi_{\alpha}^{\beta}\right\}\right)$ and $\left(T,\left\{\theta_{\alpha}\right\}\right)=\operatorname{inv} \lim \left(\left\{T_{\beta}\right\},\left\{\theta_{\alpha}^{\beta}\right\}\right)$.

(i) If for every $\alpha \in A, u_{\alpha}: C\left(S_{\alpha}\right) \rightarrow C\left(T_{\alpha}\right)$ is a bounded linear operator such that $u_{\beta} \pi_{\alpha}^{\beta \circ}=\theta_{\alpha}^{\beta \circ} u_{\alpha}$ for $\alpha \leqq \beta$ and $\sup \left\{\left\|u_{\alpha}\right\|\right\}_{\alpha \in A}<\infty$, then there is a unique linear operator $u=\operatorname{dir} \lim u_{\alpha}: C(S) \rightarrow C(T)$ such that $u \pi_{\alpha}^{\circ}=\theta_{\alpha}^{\circ} u_{\alpha}$ for all $\alpha \in A$ and $\|u\| \leqq$ $\sup \left\{\left\|u_{\alpha}\right\|\right\}_{\alpha \in A}$; if each $u_{\alpha}$ is regular, then so is $u$.

(ii) If for every $\alpha \in A, \varphi_{\alpha}: S_{\alpha} \rightarrow T_{\alpha}$ is continuous with $\varphi_{\alpha} \pi_{\alpha}^{\beta}=\theta_{\alpha}^{\beta} \varphi_{\beta}$ for $\alpha \leqq \beta$ and the $u_{\alpha}$ 's in (i) are linear exaves for the $\varphi_{\alpha}$ 's, then $u=\operatorname{dir} \lim u_{\alpha}$ is a linear exave for $\varphi=\operatorname{inv} \lim \varphi_{\alpha}: S \rightarrow T$. If each $u_{\alpha}$ is an operator of averaging (resp. extension), then so is $u$.

Proof. (i) Let $M=\bigcup\left\{\pi_{\alpha}^{\circ} C\left(S_{\alpha}\right)\right\}_{\alpha \in A}$. If $\alpha \leqq \gamma$, then $\pi_{\alpha}=\pi_{\alpha}^{\gamma} \pi_{\gamma}$ and $\pi_{\alpha}^{\circ} C\left(S_{\alpha}\right)=$ $\pi_{\gamma}^{\circ} \pi_{\alpha}^{\gamma \circ} C\left(S_{\alpha}\right) \subset \pi_{\gamma}^{\circ} C\left(S_{\gamma}\right)$. Since $A$ is directed it follows easily that $M$ is a selfadjoint subalgebra of $C(S)$ which contains the identity. Moreover, if $x \neq y$ in $S$, then 
$\pi_{\alpha}(x) \neq \pi_{\alpha}(y)$ for some $\alpha \in A$ and hence, for some $h \in C\left(S_{\alpha}\right), h \pi_{\alpha}(x) \neq h \pi_{\alpha}(y)$, i.e., $\pi_{\alpha}^{\circ}(h)(x) \neq \pi_{\alpha}^{\circ}(h)(y)$. Therefore $M$ separates the points of $S$ so that, by the StoneWeierstrass theorem, $M$ is dense in $C(S)$ and it suffices to define $u$ on $M$. Since each $\pi_{\alpha}^{\beta}$ is onto it follows that each $\pi_{\alpha}$ is onto so that the $\pi_{\alpha}^{\circ}$ 's are isometric embeddings. Our requirement that $u \pi_{\alpha}^{\circ}=\theta_{\alpha}^{\circ} u_{\alpha}$ for all $\alpha \in A$ then dictates that we define $u$ on $M$ by setting $u(f)=\theta_{\alpha}^{\circ} u_{\alpha}\left(\pi_{\alpha}^{\circ}\right)^{-1}(f)$ for $f \in \pi_{\alpha}^{\circ} C\left(S_{\alpha}\right)$. It is clear that, providing our definition makes sense, the unique extension of this $u$ to all of $C(S)$ is the one and only operator which satisfies all our requirements. Suppose then that $f=\pi_{\alpha}^{\circ}(g)=\pi_{\beta}^{\circ}(h)$, where $g \in C\left(S_{\alpha}\right), h \in C\left(S_{\beta}\right)$. Then for $\gamma \geqq$ both $\alpha$ and $\beta$,

$$
\pi_{\gamma}^{\circ} \pi_{\alpha}^{\gamma \circ}(g)=\pi_{\alpha}^{\circ}(g)=\pi_{\beta}^{\circ}(h)=\pi_{\gamma}^{\circ} \pi_{\beta}^{\gamma \circ}(h)
$$

so that $\pi_{\alpha}^{\gamma^{\circ}}(g)=\pi_{\beta}^{\gamma \circ}(h)$. Hence

$$
\begin{aligned}
\theta_{\alpha}^{\circ} u_{\alpha}\left(\pi_{\alpha}^{\circ}\right)^{-1}(f) & =\theta_{\gamma}^{\circ} \theta_{\alpha}^{\gamma \circ} u_{\alpha}(g)=\theta_{\gamma}^{\circ} u_{\gamma} \pi_{\alpha}^{\gamma \circ}(g) \\
& =\theta_{\gamma}^{\circ} u_{\gamma} \pi_{\beta}^{\gamma \circ}(h)=\theta_{\gamma}^{\circ} \theta_{\beta}^{\gamma \circ} u_{\beta}(h)=\theta_{\beta}^{\circ} u_{\beta}\left(\pi_{\beta}^{\circ}\right)^{-1}(f),
\end{aligned}
$$

which shows that $u$ is well-defined.

(ii) We must show that $\varphi^{\circ} u \varphi^{\circ}=\varphi^{\circ}$ and since $\bigcup\left\{\theta_{\alpha}^{\circ} C\left(T_{\alpha}\right)\right\}_{\alpha \in A}$ is dense in $C(T)$, it suffices to verify that $\varphi^{\circ} u \varphi^{\circ} \theta_{\alpha}^{\circ}=\varphi^{\circ} \theta_{\alpha}^{\circ}$ for all $\alpha \in A$. But

$$
\varphi^{\circ} \theta_{\alpha}^{\circ}=\left(\theta_{\alpha} \varphi\right)^{\circ}=\left(\varphi_{\alpha} \pi_{\alpha}\right)^{\circ}=\pi_{\alpha}^{\circ} \varphi_{\alpha}^{\circ}
$$

so that

$$
\varphi^{\circ} u \varphi^{\circ} \theta_{\alpha}^{\circ}=\varphi^{\circ} u \pi_{\alpha}^{\circ} \varphi_{\alpha}^{\circ}=\varphi^{\circ} \theta_{\alpha}^{\circ} u_{\alpha} \varphi_{\alpha}^{\circ}=\pi_{\alpha}^{\circ} \varphi_{\alpha}^{\circ} u_{\alpha} \varphi_{\alpha}^{\circ}=\pi_{\alpha}^{\circ} \varphi_{\alpha}^{\circ}=\varphi^{\circ} \theta_{\alpha}^{\circ} .
$$

The last assertion in (ii) merely says that inv $\lim \varphi_{\alpha}$ is onto (resp. one-to-one) whenever each $\varphi_{\alpha}$ is onto (resp. one-to-one).

Corollary 1. Let $A=\{\alpha: 0 \leqq \alpha<\Omega\}$ be an interval of ordinals and let $\left(\left\{S_{\beta}\right\},\left\{\pi_{\alpha}^{\beta}\right\}\right)$ be an inverse limit system of compact Hausdorff spaces and continuous maps over A, where each $\pi_{\alpha}^{\alpha+1}$ is onto and admits a regular linear operator of averaging. Suppose further that for every limit ordinal $\gamma<\Omega,\left(S_{\gamma},\left\{\pi_{\alpha}^{\gamma}\right\}\right)=\operatorname{inv} \lim _{\beta<\gamma}\left(\left\{S_{\beta}\right\},\left\{\pi_{\alpha}^{\beta}\right\}\right)$. Then, if $\left(S_{\Omega},\left\{\pi_{\alpha}\right\}\right)=\operatorname{inv} \lim _{\beta<\Omega}\left(\left\{S_{\beta}\right\},\left\{\pi_{\alpha}^{\beta}\right\}\right)$, the map $\pi_{0}: S_{\Omega} \rightarrow S_{0}$ is onto and admits a regular averaging operator.

Proof. Let $T_{\alpha}=S_{0}, \theta_{\alpha}^{\beta}=\mathrm{id}_{S_{0}}, \varphi_{\alpha}=\pi_{0}^{\alpha}$ for $0<\alpha \leqq \beta$ and let $u_{\alpha}^{\alpha+1}$ be a regular averaging operator for $\pi_{\alpha}^{\alpha+1}$. Inductively define $u_{\alpha}: C\left(S_{\alpha}\right) \rightarrow C\left(T_{\alpha}\right)$ for $0<\alpha<\Omega$ by: $u_{1}=u_{0}^{1}, u_{\alpha}=u_{\alpha-1} u_{\alpha-1}^{\alpha}$ if $\alpha$ is not a limit ordinal, and $u_{\alpha}=\operatorname{dir}_{\lim _{\beta<\alpha}}\left(u_{\beta}\right)$ if $\alpha$ is a limit ordinal. We note that

(a) if $\alpha$ is a limit ordinal we may identify $\left(S_{0},\left\{\operatorname{id}_{S_{0}}\right\}\right)=\left(T_{\gamma},\left\{\theta_{\alpha}^{\gamma}\right\}\right)$ with inv $\lim _{\beta<\gamma}\left(\left\{T_{\beta}\right\},\left\{\theta_{\alpha}^{\beta}\right\}\right)$ and then $\pi_{0}^{\gamma}=\operatorname{inv} \lim _{\beta<\gamma}\left(\varphi_{\beta}\right)$,

(b) the composition of regular averaging operators of a finite sequence of maps is a regular averaging operator of the composition of the maps,

(c) if

$u_{\beta}$ is well-defined and $u_{\beta} \pi_{\alpha}^{\beta \circ}=\theta_{\alpha}^{\beta \circ} u_{\alpha}=u_{\alpha}$ for $\alpha \leqq \beta<\gamma$, 
then if $\gamma$ is a limit ordinal, Lemma 2 implies that $\left(^{*}\right)$ holds for $\beta \leqq \gamma$, whereas if $\gamma$ is not a limit ordinal, then for $\alpha<\gamma$,

$$
u_{\gamma} \pi_{\alpha}^{\gamma \circ}=u_{\gamma-1} u_{\gamma-1}^{\gamma} \pi_{\gamma-1}^{\gamma \circ} \pi_{\alpha}^{\gamma-1 \circ}=u_{\gamma-1} \pi_{\alpha}^{\gamma-1 \circ}=u_{\alpha}
$$

so that again $\left(^{*}\right)$ holds for $\beta \leqq \gamma$.

It is clear then that the resulting system is well-defined and satisfies the hypotheses of Lemma 2 so that $u_{\Omega}=\operatorname{dir} \lim u_{\alpha}$ is a regular averaging operator for inv $\lim \varphi_{\alpha}=\pi_{0}$.

3. Proof of Theorem 1. Let $S$ be a compact Hausdorff space of weight $\mathfrak{m} \geqq \boldsymbol{\aleph}_{0}$. Choose a neighborhood base of the diagonal in $S \times S$ having cardinality $m$ and consisting of open symmetric sets (see [3, Chapter 6] for the terminology and notation of this paragraph). Suppose $B_{0}, B_{1}, B_{2}, \ldots$ is a well-ordering of this base where we may assume that the index set $A$ consists of all ordinals $<\Omega$, the first ordinal of cardinality $m$. Each member $B_{\alpha}$ of the base gives rise to an open cover $\left\{B_{\alpha}(s)\right\}_{s \in S}$ of $S$, where $B_{\alpha}(s)=\left\{s^{\prime} \in S:\left(s, s^{\prime}\right) \in B_{\alpha}\right\}$. For each $\alpha$ choose a finite subcover $\mathscr{U}_{\alpha}$ of $\left\{B_{\alpha}(s)\right\}_{s \in S}$. The collection $\left\{\mathscr{U}_{\alpha}\right\}$ has the following property: if $s_{1} \neq s_{2}$ in $S$, then for some $\alpha$ the closure of no member of $\mathscr{U}_{\alpha}$ contains both $s_{1}$ and $s_{2}$; for we can find $B_{\alpha_{1}}$ with $\left(s_{1}, s_{2}\right) \notin B_{\alpha_{1}}$ and then choose $B_{\alpha}$ so that $B_{\alpha} \circ B_{\alpha} \circ B_{\alpha} \circ B_{\alpha}$ $\subset B_{\alpha_{1}}$, from which the assumption that for some $s \in S$ both $s_{1}$ and $s_{2}$ are in $\left(B_{\alpha}(s)\right)^{-}$ $\subset\left(B_{\alpha} \circ B_{\alpha}\right)(s)$ gives that $\left(s_{1}, s\right)$ and $\left(s, s_{2}\right)$ are in $B_{\alpha} \circ B_{\alpha}$, hence that $\left(s_{1}, s_{2}\right)$ is in $B_{\alpha_{1}}$, a contradiction.

We proceed by induction to define an inverse limit system $\left(\left\{S_{\beta}\right\},\left\{\pi_{\alpha}^{\beta}\right\}\right)$ over $A$ satisfying the hypotheses of Corollary 1.

Define $\mathscr{V}_{n}$ for $0 \leqq n<\omega$ as follows:

$$
\mathscr{V}_{0}=\left\{U^{-}: U \in \mathscr{U}_{0}\right\}, \quad \mathscr{V}_{n}=\left\{(U \cap V)^{-}: U \in \mathscr{U}_{n}, V \in \mathscr{V}_{n-1}\right\}, \quad n \geqq 1 .
$$

We note that

(i) each $\mathscr{V}_{n}$ is a finite cover of $S$ by closed sets whose interiors cover $S$,

(ii) $\mathscr{V}_{n+1}$ refines $\mathscr{V}_{n}$, and

(iii) each member $V$ of $\mathscr{V}_{n}$ is a union of members of $\mathscr{V}_{n+1}$ whose interiors in the relative topology of $V$ cover $V$.

Now set $S_{0}=S$ and for $0 \leqq n<\omega$ let $C_{n}$ be the collection of all chains $c: V_{n}$ $\subset V_{n-1} \subset \ldots \subset V_{0}$, where $V_{i} \in \mathscr{V}_{i}, i=0,1, \ldots, n$. Given such a chain $c \in C_{n}$, let $V(c)=V_{n}$ and for $n \geqq 1$ let $c^{*} \in C_{n-1}$ be the chain $V_{n-1} \subset \ldots \subset V_{0}$. For each chain $c \in C_{n}$, let $S_{n+1, c}=V(c) \times\{c\}$, the topological product of $V(c)$ (as a subspace of $S$ ) and the one-point space $\{c\}$. Let $S_{n+1}$ be the topological sum (or union) of all the spaces $S_{n+1, c}$ for $c \in C_{n}$. Then each $S_{n+1}$ is a compact Hausdorff space of weight $\leqq \mathfrak{m}$ and the sets $S_{n+1, c}$ are both closed and open (clopen) in $S_{n+1}$.

Define $\pi_{0}^{1}: S_{1} \rightarrow S_{0}$ by $\pi_{0}^{1}(x, c)=x$, and for $n>0, \pi_{n}^{n+1}: S_{n+1} \rightarrow S_{n}$ by $\pi_{n}^{n+1}(x, c)$ $=\left(x, c^{*}\right)$. (Note that if $(x, c) \in S_{n+1, c}$ then $x \in V(c) \subset V\left(c^{*}\right)$ so that $\left(x, c^{*}\right) \in V\left(c^{*}\right)$ $\left.\times\left\{c^{*}\right\}=S_{n, c^{*}}\right)$ It is easily seen that each $\pi_{n}^{n+1}, n \geqq 0$, is a continuous surjection 
which by Lemma 1 admits a regular averaging operator (using (i), (ii), (iii) above and the fact that each $\pi_{n}^{n+1} \mid S_{n+1, c}$ is a homeomorphism onto its image).

Now let $\left(S_{\omega},\left\{\pi_{n}^{\omega}\right\}\right)=\operatorname{inv} \lim _{n<\omega}\left(\left\{S_{n}\right\},\left\{\pi_{k}^{n}\right\}\right)$, where $\pi_{k}^{n}=\pi_{k}^{k+1} \cdots \pi_{n-2}^{n-1} \pi_{n-1}^{n}$. Continue by applying the same process to $S_{\omega}$ as was applied to $S$, using now the sequence of open covers $\left(\pi_{0}^{\omega}\right)^{-1}\left(\mathscr{U}_{\omega+n}\right), n=0,1,2, \ldots$, and get $S_{\omega+1}=\left(S_{\omega}\right)_{1}$ $S_{\omega+2}=\left(S_{\omega}\right)_{2}, \ldots$ with maps $\pi_{\omega}^{\omega+1}, \pi_{\omega+1}^{\omega+2}, \ldots$ Then define $\left(S_{\omega 2},\left\{\pi_{\alpha}^{\omega 2}\right\}\right)=$ inv $\lim _{\beta<\omega 2}\left(\left\{S_{\beta}\right\},\left\{\pi_{\alpha}^{\beta}\right\}\right)$ and continue in the obvious manner.

It is clear that this process gives us a system satisfying the hypotheses of Corollary 1. Hence if we let $\left(S_{\Omega},\left\{\pi_{\alpha}\right\}\right)=\operatorname{inv} \lim _{\beta<\Omega}\left(\left\{S_{\beta}\right\},\left\{\pi_{\alpha}^{\beta}\right\}\right)$, then $\pi_{0}: S_{\Omega} \rightarrow S_{0}=S$ is a continuous surjection which admits a regular averaging operator. Since the continuous image of a compact Hausdorff space has weight $\leqq$ the weight of the space and since the product of $\mathfrak{m}$ spaces of weight $\leqq \mathfrak{m}$ has weight $\leqq \mathfrak{m}$ for $\mathfrak{m} \geqq \boldsymbol{N}_{0}$, it is clear that $S_{\Omega}$ has weight $\mathrm{m}$. We show $S_{\Omega}$ is totally disconnected by showing that any two distinct points $x, y$ in $S_{\Omega}$ can be separated by clopen sets:

Case 1. $\pi_{0}(x)=\pi_{0}(y)$. Since $x \neq y$, there is an $\alpha<\Omega$ such that $x_{\alpha} \neq y_{\alpha}$ (where we write $x_{\alpha}$ for $\left.\pi_{\alpha}(x)\right)$ and we may suppose that $\alpha$ is the first ordinal for which this is the case. Then $\alpha$ is not a limit ordinal (for a limit ordinal $\alpha, S_{\alpha}$ is by definition inv $\lim _{\beta<\alpha}\left(S_{\beta}\right)$, so that $x_{\beta}=y_{\beta}$ for all $\beta<\alpha$ implies that $\left.x_{\alpha}=y_{\alpha}\right)$. Hence $\alpha=\beta+n$, where $\beta$ is either a limit ordinal or 0 and $n$ is an integer $\geqq 1$. It follows then that $x_{\alpha}$ and $y_{\alpha}$ are in different $\left(S_{\beta}\right)_{n, c}$ 's in $S_{\alpha}=\left(S_{\beta}\right)_{n}$, for $\pi_{\alpha-1}^{\alpha}$ is one-to-one on each $\left(S_{\beta}\right)_{n, c}$ so that, if $x_{\alpha}$ and $y_{\alpha}$ were in the same $\left(S_{\beta}\right)_{n, c}$, we would have $\pi_{\alpha-1}^{\alpha}\left(x_{\alpha}\right) \neq \pi_{\alpha-1}^{\alpha}\left(y_{\alpha}\right)$, i.e. $x_{\alpha-1} \neq y_{\alpha-1}$, which contradicts our choice of $\alpha$ if $\alpha>1$ and contradicts $\pi_{0}(x)$ $=\pi_{0}(y)$ if $\alpha=1$. Hence for some $c, x$ and $y$ are separated by the clopen set $\pi_{\alpha}^{-1}\left(S_{\beta}\right)_{n, c}$.

Case 2. $\pi_{0}(x) \neq \pi_{0}(y)$. Choose $\mathscr{U}_{\alpha}$ so that the closure of no member of $\mathscr{U}_{\alpha}$ contains both $\pi_{0}(x)$ and $\pi_{0}(y)$. Then if $\alpha+1=\beta+n$, where $\beta$ is either a limit ordinal or 0 and $n$ is an integer $\geqq 1$, we must have $x_{\alpha+1}$ and $y_{\alpha+1}$ in different $\left(S_{\beta}\right)_{n, c}$ 's in $S_{\alpha+1}$, for otherwise $x_{\beta}=\pi_{\beta}^{\alpha+1}\left(x_{\alpha+1}\right)$ and $y_{\beta}=\pi_{\beta}^{\alpha+1}\left(y_{\alpha+1}\right)$ would both belong to the closure of some member of $\left(\pi_{0}^{\beta}\right)^{-1}\left(\mathscr{U}_{\alpha}\right)$ and hence $\pi_{0}(x)=\pi_{0}^{\beta}\left(x_{\beta}\right)$ and $\pi_{0}(y)=\pi_{0}^{\beta}\left(y_{\beta}\right)$ would both belong to the closure of some member of $\mathscr{U}_{\alpha}$. Hence for some $c, x$ and $y$ are separated by the clopen set $\pi_{\alpha+1}^{-1}\left(S_{\beta}\right)_{n, c}$.

Finally, if $K$ is the Cantor set, $S^{\prime}=S_{\Omega} \times K$ is a totally disconnected perfect compact Hausdorff space of weight $\mathrm{m}$, and if $\theta: S_{\Omega} \rightarrow S^{\prime}$ is any continuous section of the projection map $\pi: S^{\prime} \rightarrow S_{\Omega}$, so that $\pi \theta$ is the identity on $S_{\Omega}$, then $\theta^{\circ}: C\left(S^{\prime}\right)$ $\rightarrow C\left(S_{\Omega}\right)$ is a regular averaging operator for $\pi$. Hence for any regular averaging operator $u$ for $\pi_{0}, u \theta^{\circ}$ is a regular averaging operator for the continuous surjection $\pi_{0} \pi: S^{\prime} \rightarrow S$. This completes the proof.

REMARK. In the metric case the above proof can be simplified by taking the index set $A$ to be the natural numbers and for each $n$ in $A$ taking $\mathscr{U}_{n}$ to be any finite cover of $S$ by open sets of diameter $\leqq 1 / n$.

Let us note before proceeding that if $X$ is totally disconnected and of weight $\mathrm{m}$, then $X$ can be regarded as a closed subset of $D^{\mathfrak{m}}$. For we can find a base $B$ for the 
topology of $X$ which has cardinality $\mathfrak{m}$ and consists entirely of clopen sets and, letting $k_{U}$ be the characteristic function of $U$, the map from $X$ to $D^{B}=D^{\mathfrak{m}}$ which takes $x \in X$ into $\left(k_{U}(x)\right)_{U \in B}$ is an embedding.

COROLlARY 2. If $S$ is an absolute extensor for totally disconnected spaces, then $S$ is a Milutin space.

Proof. Let $S$ have weight $\mathfrak{m}$. Then by Theorem 1 and our remarks above, there is a closed subset $S^{\prime}$ of $D^{\mathfrak{m}}$ and a continuous surjection $\varphi_{1}: S^{\prime} \rightarrow S$ which admits a regular averaging operator $u_{1}$. Let $\varphi: D^{\mathfrak{m}} \rightarrow S$ be a continuous extension of $\varphi_{1}$ and let $\theta: S^{\prime} \rightarrow D^{\mathfrak{m}}$ be the inclusion map. Then $\varphi_{1}=\varphi \theta$ and $\operatorname{id}_{C(S)}=u_{1} \varphi_{1}^{\circ}=u_{1} \theta^{\circ} \varphi^{\circ}$ so that $u=u_{1} \theta^{\circ}$ is a regular averaging operator for $\varphi$.

4. A representation theorem for regular linear exaves. The theorem of this section is motivated by the explicit construction given by Milutin in the proof of his lemma. To give a general idea of what is involved we sketch this construction here (see [7, Lemma 5.5] for details). First, since the Cantor set $K$ is homeomorphic to $K \times K$ (both are homeomorphic to $D^{\boldsymbol{N}_{0}}$ ), $K \times K$ can be used instead of $K$. Now let $h$ be the Cantor map of $K$ onto the unit interval $I$, i.e. $h\left(\sum_{n=1}^{\infty} 2 \xi_{n} 3^{-n}\right)$ $=\sum_{n=1}^{\infty} \xi_{n} 2^{-n}$, where $\left(\xi_{n}\right) \in D^{N_{0}}$, and define $\eta: I \rightarrow K$ by $\eta(t)=\min h^{-1}(t)$. The map $\varphi: K \times K \rightarrow I$ is then defined by letting $\varphi(\xi, \zeta)$ be the nonnegative root of the equation $h(\zeta)=\lambda h(\xi)+\lambda^{2}(1-h(\xi))$ and the operator $u: C(K \times K) \rightarrow C(I)$ is obtained by setting $u f(t)=\int_{0}^{1} f(\theta(t, x)) d x$, where $\theta: I \times I \rightarrow K \times K$ is given by $\theta(t, x)=$ $\left(\eta(x), \eta\left(t x+t^{2}(1-x)\right)\right) . \varphi$ and $u$ are the required map and operator. The following theorem shows that this kind of integral representation of $u$ is a general phenomenon.

THEOREM 2. If $S$ and $T$ are compact metric spaces, a necessary and sufficient condition that $u: C(S) \rightarrow C(T)$ be a regular linear exave for a continuous map $\varphi: S \rightarrow T$ is that for $f \in C(S), t \in T$,

$$
u f(t)=\int_{0}^{1} f(\theta(t, x)) d x
$$

where $\theta: T \times I \rightarrow S$ is a function having the following properties:

(1) $\theta(t, x) \in \varphi^{-1}(t)$ for all $t \in \varphi(S), x \in I$.

(2) $\theta(t, \cdot): I \rightarrow S$ is continuous off a countable set $N_{t}$ and is left continuous everywhere, for all $t \in T$.

(3) $\theta(\cdot, x): T \rightarrow S$ is continuous at $t$ for $x \notin N_{t}$, for all $t \in T$,

Proof. (a) If such a function $\theta$ is given, then for any $t \in T, \theta(t, \cdot)$ is Borel measurable and $\theta(\cdot, x)$ is continuous at $t$ for almost all $x$ (with respect to Lebesgue measure). Hence for any $f \in C(S)$ the function $f \theta(t, \cdot)$ is integrable and the definition $u f(t)=\int_{0}^{1} f \theta(t, x) d x$ makes sense. Moreover, if the sequence $t_{n} \rightarrow t$ in $T$, then $\theta\left(t_{n}, x\right) \rightarrow \theta(t, x)$ for almost all $x$ so that $f \theta\left(t_{n}, \cdot\right) \rightarrow f \theta(t, \cdot)$ almost everywhere 
and, by Lebesgue's dominated convergence theorem, it follows that $u f\left(t_{n}\right) \rightarrow u f(t)$. Therefore $u f \in C(T)$. In addition, it is clear that $u$ is linear, $\|u\| \leqq 1$, and $u\left(1_{S}\right)=1_{T}$, so that $u$ is a regular linear operator. Finally, if $g \in C(T)$ and $s \in S$, then, by (1), $\varphi \theta(\varphi(s), x)=\varphi(s)$ for all $x$ so that

$$
\begin{aligned}
\varphi^{\circ} u \varphi^{\circ}(g)(s) & =u(g \varphi)(\varphi(s))=\int_{0}^{1} g \varphi \theta(\varphi(s), x) d x \\
& =\int_{0}^{1} g \varphi(s) d x=\varphi^{\circ}(g)(s),
\end{aligned}
$$

i.e. $u$ is a linear exave for $\varphi$.

(b) To prove the converse we may assume that $S=K$, the Cantor set. For let us suppose that the theorem has already been proved when the domain space is the Cantor set and that we are given the map $\varphi: S \rightarrow T$ with regular linear exave $u: C(S) \rightarrow C(T)$. By Theorem 5.6 of [7] (or Theorem 1 above) there is a continuous surjection $\varphi_{1}: K \rightarrow S$ which admits a regular averaging operator $u_{1}: C(K) \rightarrow C(S)$. Since $u_{1}$ is an averaging operator, it follows easily (cf. Proposition 4.4 of [7]) that $u u_{1}$ is a regular linear exave for $\varphi \varphi_{1}: K \rightarrow T$. Hence there exists a function $\theta_{1}: T \times I \rightarrow K$ satisfying the conditions

(1) $\theta_{1}(t, x) \in\left(\varphi \varphi_{1}\right)^{-1}(t)$ for all $t \in \varphi \varphi_{1}(K)=\varphi(S), x \in I$,

(2) $\theta_{1}(t, \cdot): I \rightarrow K$ is continuous off a countable set $N_{t}$ and is left continuous everywhere, for all $t \in T$,

(3) $\theta_{1}(\cdot, x): T \rightarrow K$ is continuous at $t$ for $x \notin N_{t}$, for all $t \in T$, and $u u_{1}(h)(t)=\int_{0}^{1} h \theta_{1}(t, x) d x$ for all $h \in C(K), t \in T$. If we now let

$$
\theta=\varphi_{1} \theta_{1}: T \times I \rightarrow S,
$$

then the conditions $(1)^{\prime},(2)^{\prime},(3)^{\prime}$ imply that $\theta$ satisfies (1), (2), and (3), and if $f \in C(S), t \in T$,

$$
\int_{0}^{1} f \theta(t, x) d x=\int_{0}^{1} f \varphi_{1} \theta_{1}(t, x) d x=u u_{1}\left(f \varphi_{1}\right)(t)=u\left(u_{1} \varphi_{1}^{\circ} f\right)(t)=u f(t) .
$$

Therefore, we may assume that $S=K$ and the problem is reduced to constructing the function $\theta$ for a continuous map $\varphi: K \rightarrow T$ with regular linear exave $u: C(K) \rightarrow C(T)$.

For $t \in T$, let $\mu_{t}$ be the continuous linear functional on $C(K)$ defined by $\mu_{t}(f)$ $=u f(t)$. By the Riesz representation theorem, $\mu_{t}$ may be identified with a finite regular Borel measure on $K$. Since $u$ is a regular linear exave for $\varphi$, it follows that, under this identification, $\mu_{t}$ is a nonnegative measure of total variation one which is concentrated an $\varphi^{-1}(t)$ if $t \in \varphi(K)$ (see [7, Proposition 4.1]). If $t_{n} \rightarrow t_{0}$ in $T$, then for any $f \in C(K), \mu_{t_{n}}(f) \rightarrow \mu_{t_{0}}(f)$ and, in particular, $\mu_{t_{n}}(E) \rightarrow \mu_{t_{0}}(E)$ if $E$ is a clopen subset of $K$. In what follows we shall use the standard notation [ ], ( ], etc. both for intervals in $I$ and for intervals in $K$. 
Now, for $t \in T$, let $K_{t}$ denote the support of $\mu_{t}$ and let us define the functions

$$
\begin{aligned}
\delta_{t}: K_{t} & \rightarrow I \quad \text { by } \quad \delta_{t}(\xi)=\mu_{t}[0, \xi], \\
\theta_{t}: I \rightarrow K_{t} & \text { by } \quad \theta_{t}(x)=\inf \delta_{t}^{-1}[x, 1], \text { and } \\
\theta: T \times I \rightarrow K & \text { by } \theta(t, x)=\theta_{t}(x) .
\end{aligned}
$$

Since $K_{t} \subset \varphi^{-1}(t)$ for $t \in \varphi(K), \theta$ satisfies condition (1).

Since $\mu_{t}$ is a nonnegative regular Borel measure, it is clear that

(i) $\delta_{t}$ is increasing and right continuous, for all $t \in T$, and, by the right continuity of $\delta_{t}$, it follows that

(ii) $\delta_{t} \theta_{t}(x) \geqq x$ for all $t \in T, x \in I$.

Using (i) and (ii) we proceed to establish

(iii) $\theta_{t}$ is increasing and left continuous, for all $t \in T$.

If $x_{1} \leqq x_{2}$, then $\delta_{t}^{-1}\left[x_{1}, 1\right] \supset \delta_{t}^{-1}\left[x_{2}, 1\right]$ so that $\theta_{t}\left(x_{1}\right)=\inf \delta_{t}^{-1}\left[x_{1}, 1\right] \leqq \inf \delta_{t}^{-1}\left[x_{2}, 1\right]$ $=\theta_{t}\left(x_{2}\right)$. Hence $\theta_{t}$ is increasing. To show $\theta_{t}$ is left continuous we must show that if $x_{n} \uparrow x_{0}$, so that $\theta_{t}\left(x_{n}\right) \uparrow$ some $\xi_{0} \in K_{t}$, then $\xi_{0}=\theta_{t}\left(x_{0}\right)=\inf \delta_{t}^{-1}\left[x_{0}, 1\right]$. First, $\xi_{0}$ $\leqq$ inf $\delta_{t}^{-1}\left[x_{0}, 1\right]$, for if $\xi \in \delta_{t}^{-1}\left[x_{0}, 1\right]$, then for all $n, x_{n} \leqq x_{0} \leqq \delta_{t}(\xi)$, i.e. $\xi \in \delta_{t}^{-1}\left[x_{n}, 1\right]$, so that $\theta_{t}\left(x_{n}\right) \leqq \xi$ for all $n$ and hence $\xi_{0} \leqq \xi$. Therefore it suffices to show that $\xi_{0} \in \delta_{t}^{-1}\left[x_{0}, 1\right]$ and this follows since, by (ii), $x_{n} \leqq \delta_{t} \theta_{t}\left(x_{n}\right)$ for all $n$ and hence $x_{0}=\lim _{n} x_{n} \leqq \lim _{n} \delta_{t} \theta_{t}\left(x_{n}\right) \leqq \delta_{t}\left(\xi_{0}\right)$ (since $\theta_{t}\left(x_{n}\right) \uparrow \xi_{0}$ and $\delta_{t}$ is increasing), i.e. $\xi_{0} \in \delta_{t}^{-1}\left[x_{0}, 1\right]$. This establishes (iii).

From (iii) it follows that $\theta$ satisfies condition (2).

Before verifying that condition (3) is satisfied we prove

(iv) if $x>0$, then $\theta_{t}(x) \leqq \xi$ if and only if $x \leqq \mu_{t}[0, \xi]$.

For, if $\mu_{t}[0, \xi] \geqq x>0$, then $[0, \xi] \cap K_{t} \neq \varnothing$. Letting $\xi^{\prime}=\sup \left([0, \xi] \cap K_{t}\right)$, we have $\xi \geqq \xi^{\prime} \in K_{t}, \quad \delta_{t}\left(\xi^{\prime}\right)=\mu_{t}\left[0, \xi^{\prime}\right]=\mu_{t}[0, \xi] \geqq x$, and hence, $\theta_{t}(x)=\inf \delta_{t}^{-1}[x, 1]$ $\leqq \xi^{\prime} \leqq \xi$. Conversely, if $\theta_{t}(x) \leqq \xi$, then $x \leqq \delta_{t} \theta_{t}(x)=\mu_{t}\left[0, \theta_{t}(x)\right] \leqq \mu_{t}[0, \xi]$.

To prove that $\theta$ satisfies condition (3), we must show that if $t_{0} \in T$ and $x_{0}$ is a point of continuity of $\theta\left(t_{0}, \cdot\right)=\theta_{t_{0}}$, then $\theta\left(\cdot, x_{0}\right)$ is continuous at $t_{0}$. By compactness, it suffices to show that if $t_{n} \rightarrow t_{0}$ and $\theta_{t_{n}}\left(x_{0}\right) \rightarrow \xi_{0}$, then $\xi_{0}=\theta_{t_{0}}\left(x_{0}\right)$. By including 0 and 1 in $N_{t}$, we can assume that $x_{0} \neq 0$ or 1 .

Case 1. $\theta_{t_{0}}\left(x_{0}\right)>\xi_{0}$. Then there is a $\xi$ in $K$ such that $\xi_{0} \leqq \xi<\theta_{t_{0}}\left(x_{0}\right)$ and $[0, \xi]$ is clopen. Hence, for large $n, \theta_{t_{n}}\left(x_{0}\right) \leqq \xi$ so that

$$
x_{0} \leqq \delta_{t_{n}} \theta_{t_{n}}\left(x_{0}\right)=\mu_{t_{n}}\left[0, \theta_{t_{n}}\left(x_{0}\right)\right] \leqq \mu_{t_{n}}[0, \xi] \rightarrow \mu_{t_{0}}[0, \xi]
$$

and therefore $x_{0} \leqq \mu_{t_{0}}[0, \xi]$. But, by (iv), this implies that $\theta_{t_{0}}\left(x_{0}\right) \leqq \xi$, a contradiction.

Case 2. $\theta_{t_{0}}\left(x_{0}\right)<\xi_{0}$. Then there is a $\xi$ in $K$ such that $\theta_{t_{0}}\left(x_{0}\right) \leqq \xi<\xi_{0}$ and $[0, \xi]$ is clopen. Since $x_{0}$ is a point of continuity of $\theta_{t_{0}}$ and $x_{0}<1$, there is an $x_{1}>x_{0}$ such that $\theta_{t_{0}}\left(x_{1}\right) \leqq \xi$, and since $\theta_{t_{n}}\left(x_{0}\right) \rightarrow \xi_{0}$, we eventually have $\xi<\theta_{t_{n}}\left(x_{0}\right)$ which, by (iv) gives $\mu_{t_{n}}[0, \xi]<x_{0}$. But this is impossible since we would then have

$$
x_{1} \leqq \delta_{t_{0}} \theta_{t_{0}}\left(x_{1}\right) \leqq \mu_{t_{0}}[0, \xi]=\lim _{n} \mu_{t_{n}}[0, \xi] \leqq x_{0} .
$$

Therefore $\theta_{t_{0}}\left(x_{0}\right)=\xi_{0}$ and $\theta$ satisfies condition (3). 
We must now verify that for all $f \in C(K), t \in T$,

$$
u f(t)=\mu_{t}(f)=\int_{0}^{1} f \theta(t, x) d x .
$$

However, the topology of $K$ has a base consisting of clopen sets of the form $\left(\xi_{1}, \xi_{2}\right]$ and $[0, \xi]$, and the subspace of $C(K)$ generated by the characteristic functions $k_{V}$ of such sets $V$ is dense in $C(K)$. Since $k_{\left(\xi_{1}, \xi_{2}\right]}=k_{\left[0, \xi_{2}\right]}-k_{\left[0, \xi_{1}\right]}$, this subspace is also generated by the characteristic functions of the clopen sets $[0, \xi]$. Moreover, both $u$ and the function $f \rightarrow \int_{0}^{1} f \theta(\cdot, x) d x$ are bounded linear transformations from $C(K)$ to $C(T)$ (see part (a) of this proof). Hence it suffices to verify that for $t \in T, \xi \in K$,

$$
\mu_{t}[0, \xi]=\int_{0}^{1} k_{[0, \xi]}(\theta(t, x)) d x .
$$

But, if $x>0$ and we let $A=[0, \xi] \subset K$ and $B=\left[0, \mu_{t} A\right] \subset I$, then, by (iv) above, we have $k_{A} \theta(t, x)=k_{B}(x)$, so that

$$
\int_{0}^{1} k_{A} \theta(t, x) d x=\int_{0}^{1} k_{B}(x) d x=\mu_{t}[0, \xi]
$$

This completes the proof.

\section{REFERENCES}

1. S. Eilenberg and N. E. Steenrod, Foundations of algebraic topology, Princeton Univ. Press, Princeton, N. J., 1952. MR 14, 398.

2. J. G. Hocking and G. S. Young, Topology, Addison-Wesley, Reading, Mass., 1961. MR 23 \#A2857.

3. J. L. Kelley, General topology, Van Nostrand, Princeton, N. J., 1955. MR 16, 1136.

4. E. Michael, Selected selection theorems, Amer. Math. Monthly 63 (1956), 233-238.

5. A. A. Milutin, On spaces of continuous functions, Dissertation, Moscow State University, Moscow, 1952. (Russian)

6. - Isomorphism of spaces of continuous functions on compacta having the power of the continuum, Teor. Funkciř Funkcional. Anal. i Priložen 2 (1966), 150-156. (Russian)

7. A. Pełczyński, Linear extensions, linear averagings, and their applications to linear topological classification of spaces of continuous functions, Dissertationes Math. Rozprawy Mat. 58 (1968). MR 37 \#3335.

8. Z. Semadeni, Inverse limits of compact spaces and direct limits of spaces of continuous functions, Studia Math. 31 (1968), 373-382.

Louisiana State University, Baton Rouge, Louisiana 70803 